This item was submitted to Loughborough's Research Repository by the author.

Items in Figshare are protected by copyright, with all rights reserved, unless otherwise indicated.

\title{
Dynamic interfacial fracture of a double cantilever beam
}

PLEASE CITE THE PUBLISHED VERSION

https://doi.org/10.1016/j.engfracmech.2018.11.033

PUBLISHER

(c) Elsevier

VERSION

AM (Accepted Manuscript)

PUBLISHER STATEMENT

This paper was accepted for publication in the journal Engineering Fracture Mechanics and the definitive published version is available at https://doi.org/10.1016/j.engfracmech.2018.11.033

LICENCE

CC BY-NC-ND 4.0

\section{REPOSITORY RECORD}

Chen, Tianyu, Christopher Harvey, Simon Wang, and Vadim Silberschmidt. 2018. "Dynamic Interfacial Fracture of a Double Cantilever Beam". figshare. https://hdl.handle.net/2134/36052. 


\title{
Dynamic interfacial fracture of a double cantilever beam
}

\author{
Tianyu Chen ${ }^{\mathrm{a}}$, Christopher M. Harvey, ${ }^{\mathrm{a},}$, Simon Wang, ${ }^{\mathrm{a}, \mathrm{c}}$, Vadim V. Silberschmidt ${ }^{\mathrm{b}}$ \\ ${ }^{a}$ Department of Aeronautical and Automotive Engineering, Loughborough University, \\ Loughborough, Leicestershire LE11 3TU, UK \\ ${ }^{b}$ Wolfson School of Mechanical, Electrical and Manufacturing Engineering, Loughborough University, \\ Loughborough, Leicestershire LE11 3TU, UK
}

${ }^{c}$ School of Mechanical and Equipment Engineering, Hebei University of Engineering, Handan 056038, China

\begin{abstract}
Assessment of the energy release rate (ERR) of layered material structures with account for dynamic and vibration effects is important for understanding and predicting fracture behavior in various engineering applications. In this work, the pure-mode-I interfacial fracture behavior of a symmetric double cantilever beam (DCB) under constant-rate opening displacement is studied using a dynamics and vibration analysis of Euler-Bernoulli beams, and the ERR is derived. Furthermore, a 'dynamic factor' that quantifies the dynamic effect in relation to the static component of ERR is defined. The resulting expressions are relatively short, mathematically elegant and convenient-to-use by engineers and researchers, which increases their usefulness. It is found that the dynamic factor is a function of the characteristic time only, and that this is an intrinsic property of DCB structures. An approximate method is also proposed to predict the crack extension. Predictions of ERR and crack extension are in good agreement with results from numerical results with finite-element method (FEM) simulations. Using only the first vibration mode is sufficient to capture the amplitude and frequency of ERR variation predicted by the FEM. Using higher-order vibration modes causes divergence in the amplitude of ERR oscillation; this is due to the limitation of EulerBernoulli beams in vibration analysis.
\end{abstract}

Keywords: beam dynamics; energy release rate; double cantilever beam; dynamic factor; vibration

\footnotetext{
* Corresponding Author

Email addresses: t. chen3@ lboro.ac.uk (Tianyu Chen), c.m.harvey@lboro.ac.uk (Christopher M. Harvey), s.wang@lboro.ac.uk (Simon Wang), v.silberschmidt@lboro.ac.uk (Vadim V. Silberschmidt)
} 


\begin{tabular}{|c|c|}
\hline \multicolumn{2}{|l|}{ Nomenclature } \\
\hline$A$ & Area of cross-section of beam \\
\hline$A_{0}$ & Crack area \\
\hline$b$ & Width of beam \\
\hline$E$ & Young's modulus \\
\hline$F(x)$ & Shifting function \\
\hline$f_{\mathrm{dyn}}$ & dynamic factor \\
\hline$G$ & Energy release rate \\
\hline$G_{\text {sta }}, G_{\text {dyn }}$ & Static and dynamic components of energy release rate \\
\hline$G_{c}$ & Fracture toughness \\
\hline$h$ & Thickness of beam \\
\hline$I$ & Second moment of area of beam \\
\hline$K$ & Kinetic energy \\
\hline$L$ & Length of beam \\
\hline$t$ & Time \\
\hline$U$ & Strain energy \\
\hline$v$ & Applied constant loading rate \\
\hline$w(x, t), w_{\mathrm{fv}}(x, t)$ & Total transverse deflection; transverse deflection for free vibration \\
\hline$W_{i}(x)$ & Normal mode \\
\hline$W_{\text {ext }}$ & Work done by external force \\
\hline$\Gamma$ & Energy dissipated to increase crack area \\
\hline$\eta_{i}(0), \not{\phi} \mathbb{i}_{i}(0)$ & $i$ th modal displacement and velocity \\
\hline$\rho$ & Density \\
\hline$\tau$ & Characteristic time \\
\hline$\phi_{i}(x)$ & $i$ th mode shape function \\
\hline$\omega$ & Angular frequency \\
\hline \multicolumn{2}{|l|}{ Abbreviations } \\
\hline DCB & Double cantilever beam \\
\hline ERR & Energy release rate \\
\hline FEM & Finite-element method \\
\hline VCCT & Virtual crack closure technique \\
\hline
\end{tabular}

\section{Introduction}

Composite materials have been increasingly applied over the past few decades for their superior properties over their metallic counterparts. One of the challenges with composite laminates is their propensity for interfacial delamination when subjected to various in-service conditions, including both quasi-static and dynamic loads. The former condition has received significant research attention. Dynamic fracture has, however, was considerably less studied, and is far more complicated. 
Under transient or varying external loads, the dynamic effect can become significant due to inertial and strain-rate effects. Conventionally, dynamic fracture is studied using elastodynamics, which considers the stress-wave propagation and stress superposition, or by using energy methods, which include applying a 'crack-tip energy flux integral' [1]. For an engineering composite structure, however, there seem to be few applicable analytical solutions for energy release rate (ERR) or stress intensity factor, which include dynamic effects.

The double cantilever beam (DCB) is considered to be a fundamental structure for the study of fracture behavior in layered structures. DCBs have been used on several occasions to study loading-rate effects with constant loading rates and to generate a fundamental understanding of dynamic fracture behavior. Smiley and Pipes [2] proposed a 'crack-opening displacement rate' and used this parameter to calculate the kinetic energy of a DCB. They then derived the ERR of a stationary crack and the initiation fracture toughness by assuming the same deflection as for a static beam. Blackman et al. [3] derived the ERR for a steadystate propagating crack based on the same approach. There were also experimental studies [4][5] using Smiley and Pipes' method. This method as well as Blackman et al.'s, however, provides a 'smoothed' ERR as a quadratic function with respect to time, without considering the beam vibration that leads to the oscillating ERR shown by experiments [6] and by numerical simulations [7]. Vibration may be one of the reasons why experiments used to measure initiation fracture toughness disagree on loading-rate effects [8]. Abdelmoula and Debruyne [9] investigated dynamic crack growth and arrest in a bimaterial DCB, using EulerBernoulli beams. Their theoretical model, which must be solved numerically, agrees well with the finite-element method (FEM).

In this work, the ERR of a symmetric DCB under constant-rate opening displacement is derived using a dynamics and vibration analysis of Euler-Bernoulli beams; a dynamic factor to quantify the dynamic effects is defined; and an approximate method is proposed to predict the crack extension. To the authors' knowledge, this is achieved for the first time, and the resulting expressions are relatively short, mathematically-elegant and convenient-to-use by engineers and researchers. Finally, the theory is validated against FEM simulations.

\section{Theory}

Figure 1a shows a DCB in its initial undeformed condition with its geometry, and with equal and opposite displacements $w_{0}(t)=v t$ applied to the midplane of the free ends. The 
crack tip is at the location annotated ' $\mathrm{B}$ '. It is assumed that there is no interface contact in the crack region, that parts (1) and (2) of the DCB in Figure 1a are thin and approximately classical Euler-Bernoulli beams, that the displacement are small, and that no longitudinal forces are developed. It should be noted that Euler-Bernoulli beams are inaccurate in predicting the dynamic response due to higher-order vibration modes because the phase speed increases indefinitely with increasing wave numbers [10]. Labuschagne et al. [11] proved that the eigenvalues predicted by Euler-Bernoulli theory deviate from the 2D-elastic solution very quickly with increasing mode number, and concluded that only the first two vibration modes are acceptable. In fact, for their cantilever beam configuration, they showed that the error of the second vibration mode with respect to the $2 \mathrm{D}$-elastic solution is $10.5 \%$, but the first mode is accurate. Therefore, in this study, only the first vibration mode is considered, and this is verified as accurate in determining ERR using FEM simulation results.

a)

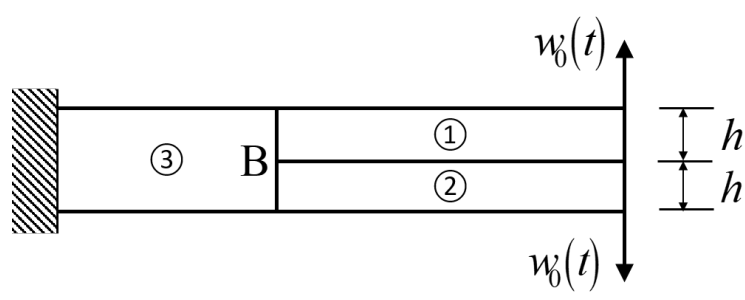

b)

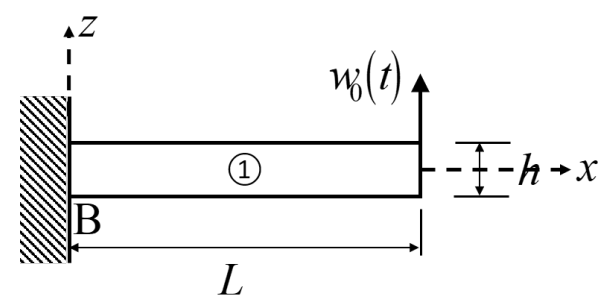

Figure 1: (a) Symmetric double cantilever beam; (b) effective boundary conditions on beam section (1)

Under the stated assumptions, beam section (1), which represents the top thin layer of the symmetric DCB, can be considered in isolation with the effective boundary conditions shown in Figure 1b. The origin of the $x$ coordinate is at the crack tip, and positive towards to the right. $L$ is variable to allow crack propagation. These effective boundary conditions are in accordance with conventional methods to determine the ERR of a symmetric DCB [12].

To determine the ERR of the DCB shown in Figure 1a, consider the conservation of energy for an elastic structure with a crack area of $A_{0}[12]$, which is

$$
\mathscr{W}_{\mathrm{ext}}^{\&}=\ell^{\&}+K^{\&}+\stackrel{\&}{ }
$$

where $W_{\text {ext }}^{\&}$ is the instantaneous power of the external forces; $U^{\&}$ and $K^{\&}$ are the changing rates of strain energy and kinetic energy respectively; and $\stackrel{\&}{\&}$ is the rate of energy dissipation due 
to the increasing crack area. Based on this energy balance, and since $\partial / \partial t=\partial A_{0} / \partial t \cdot \partial / \partial A_{0}=\AA_{0}^{\&} \cdot \partial / \partial A_{0}$, the ERR can be written as

$$
G=\frac{\partial \Gamma}{\partial A_{0}}=\frac{\partial W_{\mathrm{ext}}}{\partial A_{0}}-\frac{\partial U}{\partial A_{0}}-\frac{\partial K}{\partial A_{0}} .
$$

\subsection{Dynamic transverse response of thin beam}

Since the applied constant-rate displacement has a finite number of linearly-independent derivatives, the dynamic transverse response (deflection) of beam in Figure $1 \mathrm{~b}$ can be derived by introducing a shifting function and enforcing homogeneous conditions [13]. For constantrate displacement, $w_{0}(t)=v t$, the transverse deflection of the beam is of the form

$$
w(x, t)=w_{\mathrm{fv}}(x, t)+F(x) v t,
$$

where $w_{\mathrm{fv}}(x, t)$ represents the free vibration of the beam and $F(x)$ is the shifting function.

The governing equation for vibration of Euler-Bernoulli beams [14][15] under plane-stress conditions (in the xz plane) is

$$
E I w^{(4)}(x, t)+\rho A(x, t)=0 .
$$

For plane-strain beams, $E$ must be replaced with $E /\left(1-v^{2}\right)$. By combining Eqs. (3) and (4), and forcing homogeneous conditions, the following differential equations are obtained:

$$
\begin{gathered}
E I w_{\mathrm{fv}}^{(4)}(x, t)+\rho A(x, t)=0, \\
F^{(4)}(x)=0 .
\end{gathered}
$$

The boundary conditions for $w(x, t)$ are $w(0, t)=0, w^{(1)}(0, t)=0, w(L, t)=v t$, and $w^{(2)}(L, t)=0$. By using these boundary conditions for $w(x, t)$ in Eq. (3), and forcing homogeneous conditions, the boundary condition for the free-vibration component $w_{\mathrm{fv}}(x, t)$ and the shifting function $F(x)$ are obtained.

For the free-vibration component $w_{\mathrm{fv}}(x, t)$, the boundary conditions are $w_{\mathrm{fv}}(0, t)=0$, $w_{\mathrm{fv}}^{(1)}(0, t)=0, w_{\mathrm{fv}}(L, t)=0$ and $w_{\mathrm{fv}}^{(2)}(L, t)=0$. Note that these boundary conditions represent a fixed-pinned beam in free vibration. For the shifting function $F(x)$, the boundary conditions are $F(0)=0, F^{(1)}(0)=0, F(L)=1$ and $F^{(2)}(L)=0$. 
Beam vibration under constant-rate displacement is treated here as free vibration without any external force, with this vibration being solely excited by initial conditions. The initial conditions for free vibration $w_{\mathrm{fv}}(x, t)$ can be derived from the initial transverse deflection $w(x, 0)$ and the initial transverse velocity $\&(x, 0)$.

At $t=0$, the transverse deflection is $w(x, 0)=0$, and the transverse velocity is \& $(x, 0)=0$. These initial conditions give the initial conditions for the free vibration component as $w_{\mathrm{fv}}(x, 0)=0$ and $\&_{\mathrm{fv}}(x, t)=-F(x) v$.

The free-vibration response is the linear summation of all the modal vibrations of the beam. It can be derived by using the separation of variables method [14][15], in the form

$$
w_{\mathrm{fv}}(x, t)=\sum_{i=1}^{\infty} W_{i}(x)\left(\eta_{i}(0) \cos \omega_{i} t+\frac{\phi_{i}^{\&}(0)}{\omega_{i}} \sin \omega_{i} t\right) .
$$

In Eq. (7), $\omega_{i}=\lambda_{i}^{2} L^{-2} \sqrt{E I /(\rho A)}$, which is the angular frequency of $i$ th modal vibration; $W_{i}(x)$ represents the $i$ th normal mode, which is given by $W_{i}(x)=(\rho A L)^{-1 / 2} \phi_{i}(x)$, and $\phi_{i}(x)$ represents the mode shape, which is given by

$$
\phi_{i}(x)=\cosh \frac{\lambda_{i}}{L} x-\cos \frac{\lambda_{i}}{L} x-\sigma_{i}\left(\sinh \frac{\lambda_{i}}{L} x-\sin \frac{\lambda_{i}}{L} x\right)
$$

Solution of the frequency equation $\tan \lambda_{i}-\tanh \lambda_{i}=0$, which is derived by using the boundary conditions, determines the values of the constants $\lambda_{i}$ in Eq.(8), and then $\sigma_{i}$ is given by $\sigma_{i}=\left(\cosh \lambda_{i}-\cos \lambda_{i}\right) /\left(\sinh \lambda_{i}-\sin \lambda_{i}\right)$. Also in Eq. (7), $\eta_{i}(0)$ and $\phi_{i}(0)$ are $i$ th modal displacements and modal velocities, respectively, which are determined from the initial conditions using following equations [14]:

$$
\begin{aligned}
& \eta_{i}(0)=\int_{0}^{L} \rho A W_{i}(x) w_{\mathrm{fv}}(x, 0) d x, \\
& \oiint_{i}(0)=\int_{0}^{L} \rho A W_{i}(x) \boldsymbol{W}_{\mathrm{fv}}(x, 0) d x .
\end{aligned}
$$

The shifting function can be obtained by solving the fourth-order differential equation, $F^{(4)}(x)=0$, together with corresponding boundary conditions, which gives

$$
F(x)=-\frac{1}{2 L^{3}} x^{3}+\frac{3}{2 L^{2}} x^{2} .
$$


Note that the product of the shifting function $F(x)$ and the applied constant loading rate $v$ is the same as the 'crack opening displacement rate' in Smiley and Pipes' approach [2].

Now, combining Eqs. (7) and (11), the transverse deflection of a beam under the constant loading rate $v$ at the free end from $t=0$ is obtained as

$$
w(x, t)=v L^{2} \sqrt{\frac{\rho A}{E I}} \sum_{i=1}^{\infty} \frac{\Lambda_{i}}{\lambda_{i}^{3}} \phi_{i}(x) \sin \left(\lambda_{i}^{2} L^{-2} \sqrt{\frac{E I}{\rho A}} t\right)+\left(\frac{3}{2} L^{-2} x^{2}-\frac{1}{2} L^{-3} x^{3}\right) \cdot v t,
$$

where $\Lambda_{i}=(-1)^{i} \sqrt{\sigma_{i}^{2}+1}+\sqrt{\sigma_{i}^{2}-1}$

\subsection{Energy release rate}

The transverse deflection in Eq. (12) is now used to determine the strain energy and kinetic energy of the vibrating beam. This will allow the ERR, as given in Eq. (2), to be determined.

\subsubsection{Strain energy}

The strain energy of a beam is $U=\int_{0}^{L} M^{2}(x, t) d x /(2 E I)$, where $M(x, t)=E I w^{(2)}(x, t)$, which is the internal bending moment. The strain energy of the vibrating beam with constantrate displacement at the free end is therefore

$$
U=\frac{1}{2 E I} \int_{0}^{L}\left\{\left[E I w_{\mathrm{fv}}^{(2)}(x, t)\right]^{2}+2 E I w_{\mathrm{fv}}^{(2)}(x, t) E I F^{(2)}(x) v t+\left[E I F^{(2)}(x) v t\right]^{2}\right\} .
$$

Let $U_{1}, U_{2}$ and $U_{3}$ correspond in order to each of the three terms in Eq. (13), representing the strain energy due to localized free vibration, the strain energy due to the coupling of vibration and static motion, and the strain energy due to pure static motion (in the form of the shifting function), respectively.

The strain energy due to localized free vibration $U_{1}$ expands to 


$$
\begin{aligned}
& U_{1}=\frac{1}{2 E I} \int_{0}^{L}\left\{\left[L^{2} v \sqrt{\rho A E I} \sum_{i=1}^{\infty} \frac{\Lambda_{i}}{\lambda_{i}^{3}} \phi_{i}^{(2)}(x) \sin \left(\lambda_{i}^{2} L^{-2} \sqrt{\frac{E I}{\rho A} t}\right)\right]^{2}\right\} d x \\
& =\frac{1}{2} \rho A L^{4} v^{2} \int_{0}^{L}\left\{\left[\sum_{i=1}^{\infty} \frac{\Lambda_{i}}{\lambda_{i}^{3}} \phi_{i}^{(2)}(x) \sin \left(\lambda_{i}^{2} L^{-2} \sqrt{\frac{E I}{\rho A} t}\right)\right]^{2}\right\} d x \\
& =\frac{1}{2} \rho A L^{4} v^{2} \int_{0}^{L} \lim _{n \rightarrow \infty}\left\{\begin{array}{l}
{\left[\frac{\Lambda_{1}}{\lambda_{1}^{3}} \phi_{1}^{(2)}(x) \sin \left(\omega_{1} t\right)\right]^{2}} \\
+2 \frac{\Lambda_{2}}{\lambda_{1}^{3}} \phi_{1}^{(2)}(x) \sin \left(\omega_{1} t\right) \sum_{j=2}^{n} \frac{\Lambda_{j}}{\lambda_{j}^{3}} \phi_{j}^{(2)}(x) \sin \left(\omega_{j} t\right) \\
\left.+2 \frac{\Lambda_{2}}{\lambda_{2}^{3}} \phi_{2}^{(2)}(x) \sin \left(\omega_{2} t\right)\right]^{2} \sum_{j=3}^{n} \frac{\Lambda_{j}}{\lambda_{j}^{3}} \phi_{j}^{(2)}(x) \sin \left(\omega_{j} t\right) \\
+\mathrm{K}+\left[\frac{\Lambda_{n}}{\lambda_{n}^{3}} \phi_{n}^{(2)}(x) \sin \left(\omega_{n} t\right)\right]^{2}
\end{array}\right\} d x .
\end{aligned}
$$

Now, by applying the property of orthogonality, that is, $\int_{0}^{L} \phi_{i}^{(2)}(x) \cdot \phi_{j}^{(2)}(x) d x=\int_{0}^{L} \phi_{i}(x) \cdot \phi_{j}(x) d x=0$ with $i \neq j$, Eq. (14) simplifies to

$$
U_{1}=\frac{1}{2} \rho A L v^{2} \sum_{i=1}^{\infty} \frac{\Lambda_{i}^{2}}{\lambda_{i}^{2}} \sin ^{2}\left(\lambda_{i}^{2} L^{-2} \sqrt{\frac{E I}{\rho A}} t\right) .
$$

Eq. (15) shows that the localized free-vibration strain energy is the summation of each orthogonal vibration mode's strain energy.

Next, by expanding the strain energy term due to the coupling of local vibration and static motion, it is found to be zero (i.e. $U_{2}=0$ ). This shows that static motion doesn't alter the local vibration of this type of structure. And then, by expanding the strain-energy term due to static motion of the beam, it is found to correspond to the static strain energy due to the applied displacement, which is

$$
U_{3}=\frac{3}{2} E I L^{-3} v^{2} t^{2}
$$

The total strain energy is therefore as follows:

$$
U=\frac{1}{2} \rho A L v^{2} \sum_{i=1}^{\infty} \frac{\Lambda_{i}^{2}}{\lambda_{i}^{2}} \sin ^{2}\left(\lambda_{i}^{2} L^{-2} \sqrt{\frac{E I}{\rho A}} t\right)+\frac{3}{2} E I L^{-3} v^{2} t^{2} .
$$




\subsubsection{Kinetic energy}

The kinetic energy of the beam is

$$
K=\frac{1}{2} \rho A \int_{0}^{L}\left[\&_{\mathrm{fv}}^{2}(x, t)+2 \underset{\mathrm{fv}}{\&}(x, t) F(x) v+F^{2}(x) v^{2}\right] d x
$$

Note that $\sim_{\mathrm{fv}}(x, t)$ represents the transverse velocity of the free vibration, and that $F(x) v$ is the static motion due to the applied constant-rate displacement. In a similar way to that used before for strain energy, let $K_{1}, K_{2}$ and $K_{3}$ correspond in order to each of the three terms in Eq. (18), representing the kinetic energy due to localized vibration, the kinetic energy due to the coupling of localized vibration and static motion, and the kinetic energy due to pure static motion, respectively.

The localized vibration kinetic energy $K_{1}$ expands to

$$
\begin{aligned}
& K_{1}=\frac{1}{2} \rho A \int_{0}^{L} L_{\mathrm{fv}}^{2}(x, t) d x \\
& =\frac{1}{2} \rho A \int_{0}^{L}\left\{v \sum_{i=1}^{\infty} \frac{\Lambda_{i}}{\lambda_{i}} \phi_{i}(x) \cos \left(\lambda_{i}^{2} L^{-2} \sqrt{\frac{E I}{\rho A}} t\right)\right\}^{2} d x \\
& =\frac{1}{2} \rho A v^{2} \int_{0}^{L} \lim _{n \rightarrow \infty}\left\{\begin{array}{c}
{\left[\begin{array}{c}
\left.\frac{\Lambda_{1}}{\lambda_{1}} \phi_{1}(x) \cos \left(\omega_{1} t\right)\right]^{2} \\
+ \\
+
\end{array} \frac{\Lambda}{1}_{\lambda_{1}} \phi_{1}(x) \cos \left(\omega_{1} t\right) \sum_{j=2}^{n} \frac{\Lambda_{j}}{\lambda_{j}} \phi_{j}(x) \cos \left(\omega_{2} t\right)\right]^{2} \cos \left(\omega_{j} t\right)} \\
+2 \frac{\Lambda_{2}}{\lambda_{2}} \phi_{2} \cos \left(\omega_{2} t\right) \sum_{j=3}^{n} \frac{\Lambda_{j}}{\lambda_{j}} \phi_{j}(x) \cos \left(\omega_{j} t\right) \\
+\mathrm{K}+\left[\frac{\Lambda_{n}}{\lambda_{n}} \phi_{n}(x) \cos \left(\omega_{n} t\right)\right]^{2}
\end{array}\right\} d x .
\end{aligned}
$$

As before, by applying the property of orthogonality, Eq. (19) simplifies to

$$
K_{1}=\frac{1}{2} \rho A L v^{2} \sum_{i=1}^{\infty} \frac{\Lambda_{i}^{2}}{\lambda_{i}^{2}} \cos ^{2}\left(\lambda_{i}^{2} L^{-2} \sqrt{\frac{E I}{\rho A}} t\right) .
$$

By expanding the remaining terms, the kinetic energy due to the coupling of localized vibration and static motion is obtained as

$$
K_{2}=-\rho A L v^{2} \sum_{i=1}^{\infty} \frac{\Lambda_{i}^{2}}{\lambda_{i}^{2}} \cos \left(\lambda_{i}^{2} L^{-2} \sqrt{\frac{E I}{\rho A}} t\right),
$$


and the kinetic energy due to static motion is obtained as

$$
K_{3}=\frac{33}{280} \rho A L v^{2}
$$

Note that $K_{3}$ is the total kinetic energy used in Smiley and Pipes' [2] and Blackman et al.'s [3] work. The total kinetic energy in this work, however, is as follows:

$$
K=\rho A L v^{2}\left\{\frac{1}{2} \sum_{i=1}^{\infty} \frac{\Lambda_{i}^{2}}{\lambda_{i}^{2}} \cos ^{2}\left(\lambda_{i}^{2} L^{-2} \sqrt{\frac{E I}{\rho A}} t\right)-\sum_{i=1}^{\infty} \frac{\Lambda_{i}^{2}}{\lambda_{i}^{2}} \cos \left(\lambda_{i}^{2} L^{-2} \sqrt{\frac{E I}{\rho A}} t\right)+\frac{33}{280}\right\} .
$$

\subsubsection{Energy release rate}

The ERR of the DCB shown in Figure 1a (i.e. comprising two single beams in Figure 1b with equal and opposite displacements) is now obtained using Eq. (2) along with Eq. (17) and Eq. (23), which gives

$$
G=\frac{1}{b}\left(\begin{array}{l}
9 E I L^{-4} v^{2} t^{2}+2 \rho A v^{2} \sum_{i=1}^{\infty} \frac{\Lambda_{i}^{2}}{\lambda_{i}^{2}}\left[\cos \left(\lambda_{i}^{2} L^{-2} \sqrt{\frac{E I}{\rho A}} t\right)-1\right] \\
+4 \sqrt{\rho A E I} L^{-2} v^{2} t \sum_{i=1}^{\infty} \Lambda_{i}^{2} \sin \left(\lambda_{i}^{2} L^{-2} \sqrt{\frac{E I}{\rho A}} t\right)
\end{array}\right),
$$

in which $\sum_{i=1}^{\infty} \Lambda_{i}^{2} / \lambda_{i}^{2}=33 / 140$. Note that the ERR is proportional to the square of applied constant loading rate $v$.

Since only the first vibration mode is considered in this work, the ERR becomes

$$
G=\frac{1}{b}\left(\begin{array}{l}
9 E I L^{-4} v^{2} t^{2}+2 \rho A v^{2} \frac{\Lambda_{1}^{2}}{\lambda_{1}^{2}}\left[\cos \left(\lambda_{1}^{2} L^{-2} \sqrt{\frac{E I}{\rho A}} t\right)-1\right] \\
+4 \sqrt{\rho A E I} L^{-2} v^{2} t \Lambda_{1}^{2} \sin \left(\lambda_{1}^{2} L^{-2} \sqrt{\frac{E I}{\rho A}} t\right)
\end{array}\right) .
$$

The first term is the static component of the ERR, that is, $G_{\text {sta }}=9 E I v^{2} t^{2} /\left(b L^{4}\right)$. The remaining terms are due to dynamic effects considering the vibration of the beam. The dynamic terms can be grouped together and denoted as $G_{\text {dyn }}$, so that $G=G_{\text {sta }}+G_{\text {dyn }}$, where

$$
G_{\mathrm{dyn}}=\frac{2 v^{2}}{b}\left\{\rho A \frac{\Lambda_{1}^{2}}{\lambda_{1}^{2}}\left[\cos \left(\lambda_{1}^{2} L^{-2} \sqrt{\frac{E I}{\rho A}} t\right)-1\right]+2 \sqrt{\rho A E I} L^{-2} t \Lambda_{1}^{2} \sin \left(\lambda_{1}^{2} L^{-2} \sqrt{\frac{E I}{\rho A}} t\right)\right\} .
$$

A dynamic factor $f_{\text {dyn }}$ and a characteristic time $\tau$ are defined as, respectively, 


$$
f_{\text {dyn }}=\frac{G_{\text {dyn }}}{G_{\text {sta }}}
$$

and

$$
\tau=L^{-2} \sqrt{\frac{E I}{\rho A}} t
$$

Combining Eqs. (25) to (28) gives the dynamic factor in the following convenient form:

$$
f_{\mathrm{dyn}}=\frac{2}{9} \frac{1}{\tau^{2}} \frac{\Lambda_{1}^{2}}{\lambda_{1}^{2}}\left[\cos \left(\lambda_{1}^{2} \tau\right)-1\right]+\frac{4}{9} \frac{1}{\tau} \Lambda_{1}^{2} \sin \left(\lambda_{1}^{2} \tau\right)
$$

Based on the above definitions, the ERR is given by $G=G_{\text {sta }}\left(1+f_{\text {dyn }}\right)$. The ERR is proportional to the static component of the ERR with the ratio determined by the characteristic time only; this is an inherent property of the DCB (since $\lambda_{i}$ is determined by boundary conditions alone). Note that $\lambda_{1} \approx 3.9266$.

\subsubsection{Properties of dynamic factor}

The variation of dynamic factor $f_{\text {dyn }}$ with characteristic time $\tau$, as described by Eq. (29), is shown by the solid line in Figure 2.

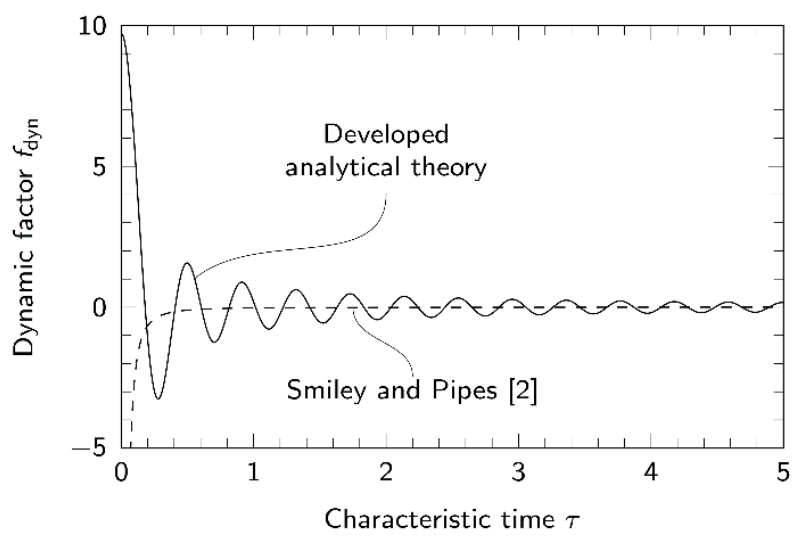

Figure 2: Dynamic factor versus characteristic time

Figure 2 shows that the dynamic factor is less than -1 during parts of the first two vibration periods, which leads to negative ERR. This finding is consistent with the previous studies of Smiley and Pipes [2] and Blackman et al. [3], although they reported infinite negative ERR at $t=0$, whereas in this work, ERR is always finite. According to fracture mechanics, negative ERR impedes crack propagation [16], because if ERR is negative, then 
crack growth increases the potential energy of the cracked solid, rather than decreasing it [17].

The dynamic factor attenuates significantly with respect to the characteristic time. At $\tau=0$ , the dynamic effect is at its maximum. The limit of $f_{\text {dyn }}$ at $\tau=0$ is

$$
\lim _{\tau \rightarrow 0} f_{\text {dyn }}(\tau)=\frac{1}{3} \lambda_{1}^{2} \Lambda_{1}^{2} \approx 9.721
$$

The dynamic factor, however, decays to $-1.0<f_{\text {dyn }}<1.0$ after one characteristic time period. It then continues to drop steadily. After around 10 characteristic time periods, the dynamic effect reduces to $-0.1<f_{\text {dyn }}<0.1$, which can be regarded as insignificant. Note that the dynamic ratio is independent of applied opening rate and that this is an intrinsic property of this type of structure.

\subsubsection{Comparison with Smiley and Pipes [2]}

Smiley and Pipes' [2] approach gives the ERR as

$$
G=\frac{1}{b}\left(9 E I L^{-4} v^{2} t^{2}-\frac{33}{140} \rho A v^{2}\right)
$$

This ERR only considers the transverse static motion along the beam regardless of the kinetic energy and strain energy induced by the beam vibration. It also predicts a negative singular ERR at $t=0$.

Note that Smiley and Pipes' 'crack opening displacement rate' corresponds to the product of the shifting function and constant loading rate in this work Therefore, the crack opening displacement rate effect was still included in this work.

Smiley and Pipes also obtained the dynamic factor as a function of the characteristic time

$\tau$ only, that is, $f_{\text {dyn }}=-11 \tau^{-2} / 420$. Smiley and Pipes' dynamic factor is shown by the dashed line in Figure 2 for comparison.

\subsection{Crack propagation}

Consider a steadily propagating crack with $G=G_{c}=$ constant and assume that the contribution to strain energy and kinetic energy due to the speed of crack propagation is small. Some works, for example Ref. [18], report that fracture toughness depends on crack propagation speed, but this is not considered in this work. The crack propagation speed can be approximately determined as 


$$
\frac{d L}{d t}=-\frac{\partial\left(G-G_{c}\right) / \partial t}{\partial\left(G-G_{c}\right) / \partial L}=-\frac{\partial G / \partial t}{\partial G / \partial L}=\frac{L}{2 t} .
$$

In evaluating the terms $\partial G / \partial t$ and $\partial G / \partial L$ based on Eq. (25), and substituting them into Eq. (32), all of the oscillatory terms cancel out, and consequently the crack propagation rate shows no oscillatory behavior. It should be remembered that Eq. (32) only applies under the stated conditions, namely, steady and slow crack propagation without contact. Physically it means that during crack propagation, the time-oscillation of the ERR is balanced by the gradient of ERR.

Note that the crack propagation speed obtained in Eq. (32) is the same as that obtained for a DCB under quasi-static loads [3]. Nevertheless, it was derived using the theory developed above for dynamic interfacial fracture and is therefore also valid for dynamic crack propagation under the stated assumptions and limitations. Eq. (32) gives the crack extension as

$$
L(t)=C_{0} \sqrt{t},
$$

where $C_{0}$ is a coefficient that can be determined using the condition that $G=G_{c}$ when $t=t_{0}$, which is the time when the crack starts to propagate.

Eq. (33) is only applicable to brittle materials with a moderate material density. If the material density is high, inertia effects can cause the crack surfaces to close and there will be crack arrest. The theory, however, cannot predict crack arrest for two reasons: (1) The condition used in deriving Eq. (33) is that $G=G_{c}$ at all times after crack initiation, meaning that the crack must always propagate. (2) The theory does not consider contact between crack surfaces, and furthermore, interpenetration of crack surfaces gives non-zero ERR. For materials with higher density, Eq. (33) can still accurately predict the slope of the crack extension-time curve.

\section{Finite-element method verification}

\subsection{Finite-element method verification for energy release rate}

To verify the analytical solution for ERR in Section 2, the symmetric DCB in Figure 3 was considered. The width of the DCB is $1 \mathrm{~mm}$. An isotropic elastic material was used with the Young's modulus of $10 \mathrm{GPa}$, Poisson's ratio of 0.3 , and density of $10^{3} \mathrm{~kg} \mathrm{~m}^{-3}$. 


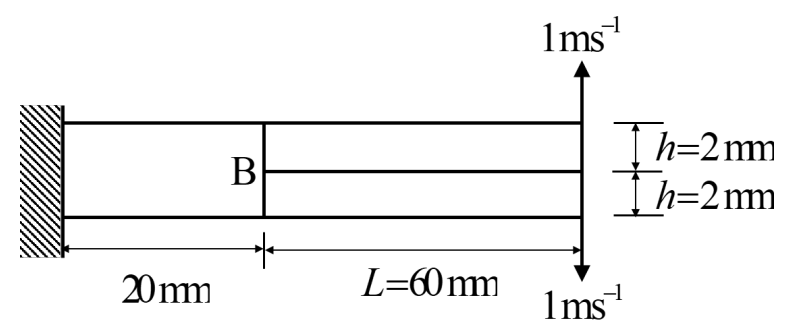

Figure 3: Geometry of DCB for numerical verification

A 2D FEM model was built using plane stress elements (CPS4R) in Abaqus/Explicit, which includes the inertia effects. In total, 32000 elements were used to simulate the DCB specimen. The damping ratio was set to zero. The virtual crack-closure technique (VCCT) was used to determine the ERR numerically. No contact model was used. The FEM results are compared with the developed analytical theory in Figure 4.

(a)

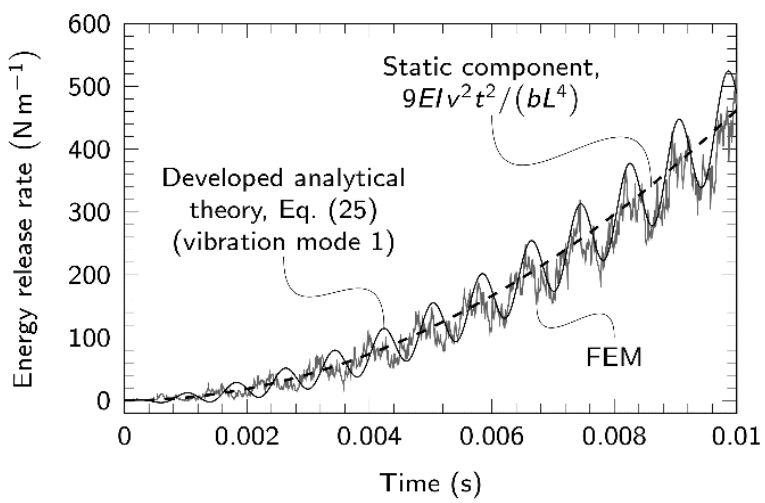

(b)

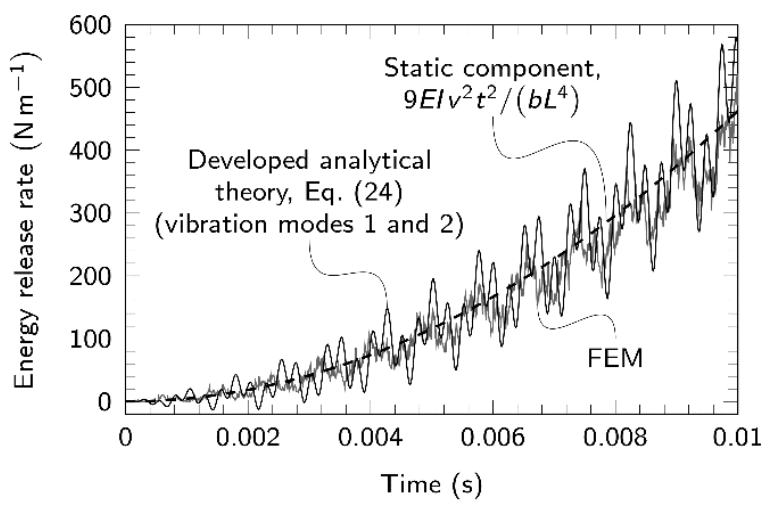

Figure 4: Comparison of energy release rate from FEM simulation and from the developed analytical theory with first vibration mode (a), and with first two vibration modes (b).

The results based on the developed analytical approach with the first vibration mode are in good agreement with the results from the numerical simulation: the analytical results capture the amplitude and frequency of ERR variation predicted by the FEM. The analytical theory is slightly out-of-phase with the FEM, which is due to the difference in boundary conditions: the FEM model simulates a full DCB, whereas the theory models the effective boundary conditions shown in Figure $1 \mathrm{~b}$.

It is worth noticing that the ERR with dynamic effects oscillates about a mean value - the static ERR. The oscillation amplitude is well predicted by the product of the dynamic factor and static ERR. In Eq. (29), the dynamic factor decays quickly with time, but in Figure 4, the oscillation amplitude actually increases with time. This indicates that the dominant contribution to this vibration amplitude is the increasing static component of ERR. 
When the first two vibration modes are included (Figure 4b), the amplitude of ERR oscillation begins to diverge: this is due to the limitation of Euler-Bernoulli beams in vibration analysis, where higher-order modes work in the same way as the lower-order modes in determining the ERR. Consequently, Euler-Bernoulli beams show a dispersive property and stress wave speed approaches to infinity with increasing natural frequency. This could be alleviated by including rotational and/or shear effects [19], but this is beyond the scope of this manuscript, and the lower-order modes are sufficient for this work.

\subsection{Finite-element method verification for crack propagation}

For crack propagation, the same geometry and material properties were used, but the fracture toughness of the material was set to $200 \mathrm{~N} \mathrm{~m}^{-1}$, and the width of the specimen set to $0.05 \mathrm{~mm}$. The crack extension was calculated using both the developed analytical theory and FEM simulation in conjunction with the VCCT and without modeling contact between the crack surfaces. Both results are plotted versus time in Figure 5 for comparison. Note that the FEM simulations in this verification exercise used 128000 3D stress elements (C3D8R) with one element in the widthwise direction. Widthwise displacement was constrained, which simulated a plane-strain condition. Consequently, the effective Young's modulus of $E /\left(1-v^{2}\right)$ was used in the analytical calculations.

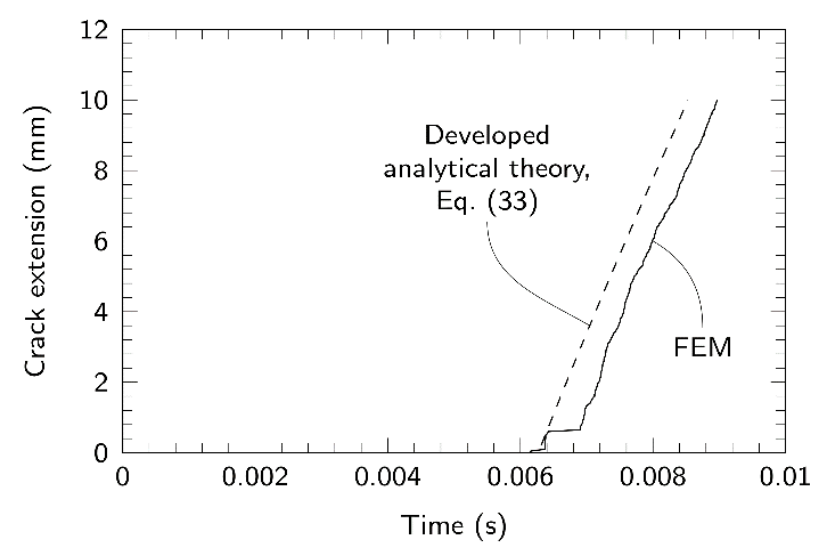

Figure 5: Comparison of crack extension obtained from FEM simulation and from developed analytical theory

Both analytical and numerical methods predict the same crack initiation time, and they also agree well with each other for the period just following crack initiation. This is as expected, since the theory has already been shown to agree well with the FEM in predicting the ERR. Subsequently, however, the FEM shows a period of crack arrest of about $0.0005 \mathrm{~s}$ 
after crack initiation, after which the crack grows steadily with a slope that is well predicted by the theory. The FEM captures the crack arrest period, but the analytical theory does not for the reasons explained in Section 2.3.

This comparison shows that, for a brittle material with moderate density, the analytical method proposed in this study can predict the ERR well (and thus the crack initiation time), as well as the slope of crack-extension curve.

\section{Conclusions}

The ERR for a symmetric DCB configuration under constant-loading-rate displacement was derived accounting for the dynamics and vibration effects. The corresponding dynamic factor and characteristic time were also defined. Furthermore, an approximate method was proposed to predict the crack extension.

Predictions of ERR are in good agreement with results from 2D FEM simulations. Using only the first vibration mode was adequate to capture the amplitude and frequency of ERR variation predicted by the FEM. Using higher-order vibration modes causes divergence in the amplitude of ERR oscillation; this is due to the limitation of Euler-Bernoulli beams in vibration analysis. For crack extension, the agreement is also good in terms of crack initiation time and crack propagation speed, but crack arrest cannot be captured. This will be exacerbated for high-density materials, which have increased inertia effects.

The dynamic factor, which quantifies the dynamic effect in relation to the static component of ERR, was shown to be a function of the characteristic time only. This is an intrinsic property of DCB structures.

To the authors' knowledge, this work presents these expressions, which are relatively short, mathematically-elegant and convenient-to-use, for the first time. They are not restricted to any particular application, and are expected to be useful to both engineers and researchers.

\section{References}

[1] L. B. Freund, Dynamic Fracture Mechanics. 1990.

[2] A. J. Smiley and R. B. Pipes, "Rate effects on mode I interlaminar fracture toughness in composite materials," Journal of Composite Materials, vol. 21, no. 7, pp. 670-687, 1987.

[3] B. R. K. Blackman, A. J. Kinloch, Y. Wang, and J. G. Williams, "The failure of fibre composites and adhesively bonded fibre composites under high rates of test," Journal 
of Materials Science, vol. 31, no. 17, pp. 4451-4466, 1996.

[4] S. I. Thorsson, A. M. Waas, J. Schaefer, B. Justusson, and S. Liguore, "Effects of elevated loading rates on mode I fracture of composite laminates using a modified wedge-insert fracture method," Composites Science and Technology, vol. 156, pp. 3947, 2018.

[5] G. Hug, P. Thévenet, J. Fitoussi, and D. Baptiste, "Effect of the loading rate on mode I interlaminar fracture toughness of laminated composites," Engineering Fracture Mechanics, vol. 73, no. 16, pp. 2456-2462, 2006.

[6] H. Liu, H. Nie, C. Zhang, and Y. Li, "Loading rate dependency of Mode I interlaminar fracture toughness for unidirectional composite laminates," Composites Science and Technology, vol. 167, pp. 215-223, Oct. 2018.

[7] Y. Liu, F. P. der Meer, and L. J. Sluys, "Cohesive zone and interfacial thick level set modeling of the dynamic double cantilever beam test of composite laminate," Theoretical and Applied Fracture Mechanics, 2018.

[8] C. Sun and C. Han, "A method for testing interlaminar dynamic fracture toughness of polymeric composites," Composites Part B: Engineering, vol. 35, no. 6-8, pp. 647$655,2004$.

[9] R. Abdelmoula and G. Debruyne, "Modal analysis of the dynamic crack growth and arrest in a DCB specimen," International Journal of Fracture, vol. 188, no. 2, pp. 187-202, 2014.

[10] S. M. Han, H. Benaroya, and T. Wei, "Dynamics of transversely vibrating beams using four engineering theories," Journal of Sound and Vibration, vol. 225, no. 5, pp. 935988, 1999.

[11] A. Labuschagne, N. F. J. van Rensburg, and A. J. van der Merwe, "Comparison of linear beam theories," Mathematical and Computer Modelling, vol. 49, no. 1-2, pp. 20-30, 2009.

[12] E. E. Gdoutos, Fracture Mechanics. 2005.

[13] D. A. Grant, "Beam vibrations with time-dependent boundary conditions," Journal of Sound and Vibration, vol. 89, no. 4, pp. 519-522, 1983.

[14] S. S. Rao, Vibration of Continuous Systems. 2007.

[15] R. D. Blevins, Formulas for Natural Frequency and Mode Shape. 1979.

[16] S. B. Park and C. T. Sun, "Effect of electric field on fracture of piezoelectric ceramics," International Journal of Fracture, vol. 70, no. 3, pp. 203-216, 1995.

[17] V. Govorukha, M. Kamlah, V. Loboda, and Y. Lapusta, Fracture Mechanics of 
Piezoelectric Solids with Interface Cracks. Springer, 2017.

[18] F. Zhou, J. F. Molinari, and T. Shioya, "A rate-dependent cohesive model for simulating dynamic crack propagation in brittle materials," Engineering Fracture Mechanics, vol. 72, no. 9, pp. 1383-1410, 2005.

[19] J. L. Guyader, Vibration in Continuous Media. Wiley, 2013. 